\title{
Correction to: COVID-19 pandemic preparation: using simulation for systems- based learning to prepare the largest healthcare workforce and system in Canada
}

Mirette Dubé ${ }^{\prime *}$, Alyshah Kaba ${ }^{1,2}$, Theresa Cronin ${ }^{1}$, Sue Barnes ${ }^{1}$, Tara Fuselli ${ }^{1}$ and Vincent Grant ${ }^{1,3,4}$

\section{Correction to: Adv Simul 5, 22 (2020) \\ https://doi.org/10.1186/s41077-020-00138-w}

The original article [1] contains copyediting errors in Table 3. The correct presentation of Table 3 can be viewed ahead in this Correction article.

\begin{abstract}
Author details
'eSIM Provincial Simulation Program, Alberta Health Services, Alberta Health Services, 1403 29th Street NW, Calgary, Alberta T2N 2 T9, Canada.

${ }^{2}$ Department of Community Health Sciences, Cumming School of Medicine, University of Calgary, Calgary, Canada. ${ }^{3}$ Departments of Pediatrics and Emergency Medicine, Cumming School of Medicine, University of Calgary, Calgary, Canada. ${ }^{4}$ KidSIM Pediatric Simulation Program, Alberta Children's Hospital, 28 Oki Dr NW, Calgary, Alberta T3B 6A8, Canada.
\end{abstract}

Published online: 20 April 2021

\section{Reference}

1. Dubé M, et al. COVID-19 pandemic preparation: using simulation for systems-based learning to prepare the largest healthcare workforce and system in Canada. Adv Simul. 2020;5:22. https://doi.org/10.1186/s41077-02000138-w.

The original article can be found online at https://doi.org/10.1186/s41077020-00138-w.

* Correspondence: mirette.dube@ahs.ca

1 eSIM Provincial Simulation Program, Alberta Health Services, Alberta Health Services, 1403 29th Street NW, Calgary, Alberta T2N 2 T9, Canada

Full list of author information is available at the end of the article

(c) The Author(s). 2021 Open Access This article is licensed under a Creative Commons Attribution 4.0 International License, which permits use, sharing, adaptation, distribution and reproduction in any medium or format, as long as you give appropriate credit to the original author(s) and the source, provide a link to the Creative Commons licence, and indicate if changes were made. The images or other third party material in this article are included in the article's Creative Commons licence, unless indicated otherwise in a credit line to the material. If material is not included in the article's Creative Commons licence and your intended use is not permitted by statutory regulation or exceeds the permitted use, you will need to obtain permission directly from the copyright holder. To view a copy of this licence, visit http://creativecommons.org/licenses/by/4.0/ The Creative Commons Public Domain Dedication waiver (http://creativecommons.org/publicdomain/zero/1.0/) applies to the data made available in this article, unless otherwise stated in a credit line to the data. 
Table 3 Key themes and qualitative outcomes (highest impact and highest frequency) identified across simulations

Key Themes and Qualitative Outcomes (Highest Impact and Highest Frequency) identified in Simulation

1. Theme: Safe doffing (removal of PPE safely and in correct order)

\section{Key Outcomes:}

- Cross monitor team members during doffing

- Use an IPAC poster as a cognitive aid

- Ensure "1 to 1" doffing to avoid breaches observed when too many doffing at once

(e.g. getting ahead or behind in doffing sequence)

- Consistent role of a "PPE Coach" to support safe doffing- ensure focus and intention with every step

- Implement "just-in-time" review of safe doffing to reduce cognitive load during long stressful periods in PPE.

\section{Theme: Conducting environmental scans of care areas is crucial in anticipating, planning ahead and developing area processes}

\section{Key Outcomes:}

- Remove visitor chairs, extra equipment and linens from room to avoid waste and additional cleaning between patients

- Keep transport routes clear

- Post signage for direction and decrease of clutter

- Creation of supply restocking checklist

- Creation of COVID-19 specific cart of required supplies

- Creation of small, labelled packages of specific supplies or medications for fast grab and go

- Ensure team members are aware of the responsibilities required to maintain the space

- Ensure cleaning processes for removal of equipment leaving COVID-19 rooms (e.g stretchers, wheelchairs)

\section{Theme: Conduct Inter-Departmental/Inter-Hospital Transport routes to establish communication} and process between departments and professions

\section{Key Outcomes:}

- Test and walk through the route

- Use signage if COVID-19 routes differ from usual process

- Clean hallways of clutter and reduce traffic if possible

- Consider dedicating elevator banks for COVID-19 patients, staff and carts

- Establish a designated clean person on transports to ensure surfaces are cleaned (e.g. floors, elevator buttons, stretchers, wheel chairs, etc.)

- Emergency Medical Services should use a common pager Stem: "Possible/Confirmed COVID-19 patient"

- Upon arrival of out of hospital emergency medical services, ensure transport is ready and routes are prepared.

\section{Theme: Maintenance of Isolation Environment/Prevention of Contamination}

\section{Key Outcomes:}

- Removal of stethoscopes, phones, ID badges, lanyards, watches, and earrings from person prior to donning.

- When items are on person, reinforce learnings re: don't reach below gown for ID badge/pager/mobile phone; or under visor to adjust goggles/mask.

- Creation of bins on an external cart in donning area for dropping items into

- Keep numbers of staff in the room low when possible

- Ensure cleaning process for roving items such as clipboards, ultrasound machines, etc.

\section{Theme: Roles and Responsibilities}

\section{Key Outcomes:}

- A runner role is needed across multi areas Operating Room, Emergency Department, Labour \& Delivery Unit, Intensive Care Unit (team member to bring supplies between isolated COVID-19 care area and non-isolated area)

- Consider the involvement of HCAs and Unit Clerks to bring necessary equipment required for teams

- Establish "clean" and "dirty" sides between rooms and within rooms by taping the floors for a visual cue

- Establish CODE COVID-19 team to attend to all rapid deteriorating patients
Systems Categories

People/Teams/Tasks; Tools/Technology;

Environment Tools/Technology; People/Teams/Tasks

People/Teams/Tasks; Environment; Tools/Technology

Tools/Technology: People/Teams/Tasks

People/Teams/Tasks Environment 
Table 3 Key themes and qualitative outcomes (highest impact and highest frequency) identified across simulations (Continued)

Key Themes and Qualitative Outcomes (Highest Impact and Highest Frequency) identified in Simulation

6. Theme: Innovative Approaches to Communication

\section{Key Outcomes:}

- Use of dry erase markers on the shared glass walls between isolation to ante room

- Use of a laminated page that can be flipped back and forth

- Use of white boards to communicate key messages to outside team members

- Use of two-way radios (e.g. walkie talkies) or baby monitors

- Use of speaker phone setting

- Use of tape on floor to communicate 'clean versus dirty' zones

- Check that monitors and speakers on phones (especially with PPE on) can be heard

- Include name/role tag stickers on outer PPE to ensure role clarity and effective communication

- Reduce noise and ensure use of closed loop communication (additional communication challenges with PPE on)

- Use of trigger scripts on pagers to signal a priority response. Scripts like "COVID airway" or "COVID transport" to alert a team and get the right people and the right equipment to the right place.

\section{Theme: Psychological Safety and Speaking Up}

\section{Key Outcomes:}

- Use critical language when breeches in PPE or when overcrowding in rooms occur

- Encourage all team members to speak up when they see breaches in safe PPE practices

- Removing hierarchical barriers can be challenging; promoting psychology safety is important for a cohesive team

- Go beyond your own professional role to cross teach about PPE

\section{Theme: Critical Care Medicine Pre-Intubation Cognitive Aid}

\section{Key Outcomes:}

- Communicate a plan to ensure staff know their roles during intubation

- Double-check proper PPE during intubation

- Most experienced practitioner should perform the intubation

- Ensure the ventilator and video laryngoscopy device are in the room prior to start

- Consider back-up plan depending on available resources

- Ensure correct bagger filter is attached

\section{Theme: Use of Cognitive Aids and Checklists}

\section{Key Outcomes}

- Consider human factors science in the development of new COVID-19 cognitive aids and checklists

- Cognitive aids can be made into posters, use larger font, central point of reference

- They should be clear, easy to use, adaptable to context, staff trained on prior to implementation and pilot tested prior to use on a real patient

- Examples: COVID-19 Airway pause checklist, checklists for buckets and carts/bins, IPAC Donning \& Doffing Poster
Systems Categories

Tools/Technology

People/Teams/Tasks

People/Teams/Tasks

People/Teams/Tasks; Tools/Technology; Organization 